\title{
Candidate gene resequencing to identify rare, pedigree-specific variants influencing healthy aging phenotypes in the long life family study
}

Todd E. Druley 1,2, Lihua Wang ${ }^{1,3}$, Shiow J. Lin ${ }^{1,3}$, Joseph H. Lee ${ }^{4,5,6}$, Qunyuan Zhang ${ }^{1,3}$, E. Warwick Daw ${ }^{1,3}$, Haley J. Abel ${ }^{1,3}$, Sara E. Chasnoff ${ }^{1,2}$, Enrique I. Ramos ${ }^{1,2}$, Benjamin T. Levinson ${ }^{1,2}$, Bharat Thyagarajan', Anne B. Newman ${ }^{8}$, Kaare Christensen ${ }^{9}$, Richard Mayeux ${ }^{10}$ and Michael A. Province ${ }^{1,3^{*}}$

\begin{abstract}
Background: The Long Life Family Study (LLFS) is an international study to identify the genetic components of various healthy aging phenotypes. We hypothesized that pedigree-specific rare variants at longevity-associated genes could have a similar functional impact on healthy phenotypes.

Methods: We performed custom hybridization capture sequencing to identify the functional variants in 464 candidate genes for longevity or the major diseases of aging in 615 pedigrees (4,953 individuals) from the LLFS, using a multiplexed, custom hybridization capture. Variants were analyzed individually or as a group across an entire gene for association to aging phenotypes using family based tests.
\end{abstract}

Results: We found significant associations to three genes and nine single variants. Most notably, we found a novel variant significantly associated with exceptional survival in the 3' UTR OBFC1 in 13 individuals from six pedigrees. OBFC1 (chromosome 10) is involved in telomere maintenance, and falls within a linkage peak recently reported from an analysis of telomere length in LLFS families. Two different algorithms for single gene associations identified three genes with an enrichment of variation that was significantly associated with three phenotypes (GSK3B with the Healthy Aging Index, NOTCH1 with diastolic blood pressure and TP53 with serum HDL).

Conclusions: Sequencing analysis of family-based associations for age-related phenotypes can identify rare or novel variants.

Keywords: Genomics, Aging, Genetics, Geriatrics, Pedigrees, Family, Sequencing

\section{Background}

According to United Nations World Population Prospects 2012 revision (http://esa.un.org/unpd/wpp/Documenta tion/pdf/WPP2012_HIGHLIGHTS.pdf), the worldwide average human lifespan was 71 years (68.5 years for males and 73.5 years for females) over the period 2010-2013. At least $25 \%$ of human lifespan is dictated by genetic factors,

\footnotetext{
* Correspondence: mprovince@wustl.edu

${ }^{1}$ Center for Genome Sciences and Systems Biology, Washington University School of Medicine, 660 South Euclid Avenue, Campus Box 8116, St. Louis, MO 63108, USA

${ }^{3}$ Division of Statistical Genomics, Department of Genetics, Washington University School of Medicine, St. Louis, MO, USA

Full list of author information is available at the end of the article
}

most of which is unknown [1]. Currently, most human longevity and healthy aging related variants identified through genome wide association study (GWAS) are either intergenic or intronic with weak effects, and there is little characterization of coding variants that may influence human lifespan. For instance, common variants in multiple genes such as apolipoprotein E $(A P O E)$ [2-5], Forkhead Box O1 (FOXO1) [6], Forkhead Box O3 (FOXO3) [7], Insulin-Like Growth Factor 1 Receptor $(I G F-1 R)[6,8]$ and Translocase of Outer Mitochondrial Membrane 40 Homolog (TOMM40) [9] have all been associated with human lifespan. However, lifespan is a highly complex trait and healthy aging is controlled and 
influenced by variety of environmental factors and individual phenotypes [10]. Leukocyte telomere length, Body Mass Index (BMI), blood pressure, serum lipids, blood glucose, cognitive function, onset of type 2 diabetes, heart disease, cancer, and stroke are a few phenotypes associated with aging, and we hypothesized that pedigree-specific coding variants associated with these traits would identify additional genes or pathways important for regulating human lifespan. While noncoding variants may have significant gene or epigenetic regulatory effects, exonic variants would be expected to exert larger effects on gene function and might therefore explain a larger fraction of the variance in these complex aging phenotypes.

Thus, we expect families clustered for long-lived healthy members $[11,12]$ to be enriched for genetic variants that will promote healthy and/or prolonged aging. To identify new, rare, heritable variants that are associated with multiple healthy aging phenotypes, we performed custom hybridization capture sequencing of 464 healthy aging related candidate genes in Phase 1 of the Long Life Family Study (LLFS). We then performed association analyses of both rare $(<1 \%$ minor allele frequency) and common variants against multiple aging-related phenotypes including survival, leukocyte telomere length, healthy aging index [13], BMI, blood pressure, blood lipid levels, blood glucose level, cognitive function, onset of type 2 diabetes, heart disease, cancer, and stroke.

\section{Methods}

\section{Subjects}

The LLFS [11], a multi-center family-based cohort study, enrolled 4,953 individuals from 539 families clustered for exceptional survival to identify environmental and genetic factors that account for the long healthy lives in these families. Phase 1 of the LLFS was conducted between 2006 and 2009. All participants provided written informed consent prior to inclusion in the study. The four recruitment centers include Boston University Medical Center, Columbia University Medical Center, University of Pittsburgh, and University of Southern Denmark. Using the Family Longevity Selection Score (FLoSS) [14], a score generated according to birth-year cohort survival probabilities of the proband and siblings, probands and their families with FLoSS score of 7 or higher, at least one living sibling, and at least one living offspring (minimum family size of 3), who were able to give informed consent and willing to participate were recruited. The spouses were enrolled as controls for this study. The characteristics of the LLFS population by generation and by gender are listed in Additional file 1: Table S1 and $\mathrm{S} 2$, respectively.

\section{Phenotype measurements}

In this study, human lifespan and phenotypic traits related to healthy aging including survival, leukocyte telomere length, BMI, blood pressure, blood lipid levels, blood glucose and insulin levels, cognitive function, healthy aging index, as well as the ages of onset of stroke, coronary heart disease, congestive heart failure, cancer, and type 2 diabetes mellitus, were investigated.

\section{Survival and mortality-weighted healthy aging index}

The lifespan in LLFS was estimated as the duration of survival free of death from any cause or from any one of a specified list of diseases including cardiovascular disease, congestive heart failure, cancer, stroke, and Type 2 diabetes. The LLFS proband generation, including probands and full and half siblings $(n=810)$, was used for identifying variants related with survival.

Healthy aging was established by the lack of disease and clinical test values indicating normal function of various biological systems. To increase the probability of identifying healthy aging-related genetic variants, which might influence one or more components of these biological systems, Sanders et al. proposed the Healthy Aging Index (HAI) as a subphenotype of longevity [13]. Using approximate age-adjusted tertiles for systolic blood pressure, pulmonary vital capacity, creatinine, fasting glucose, and Modified Mini-Mental Status Examination score, each individual is scored 0 , 1 , or 2 for each trait. The HAI is then calculated from the sum of these scores in a range from 0 (healthy) to 10 (unhealthy). The mortality-weighted HAI is generated from the sum of the mortality-weighted scores of the five components listed above. The weights are based on the regression coefficients from the Cox proportional hazards model for the effect of each component on survival and are 0.17085 for systolic blood pressure, 0.38386 for forced vital capacity, 0.42873 for MMSE points, 0.13397 for serum creatinine, and 0.23880 for serum fasting glucose.

\section{Glycated hemoglobin $\left(\mathrm{HbA}_{1 \mathrm{c}}\right)$, Blood Glucose and Insulin Levels, Leukocyte Telomere Length, Blood Lipid Levels Including Total Cholesterol, HDL Cholesterol, LDL Cholesterol and Triglyceride, BMI, Blood Pressure, Cognitive Function, Stroke, Coronary Heart Disease, Congestive Heart Failure, Cancer, Type 2 Diabetes}

We looked for any possible association between our candidate genes related to healthy aging and various aging phenotypes including $\mathrm{HbA}_{1 \mathrm{c}}$, blood glucose and insulin levels, leukocyte telomere length, blood lipid levels including total cholesterol, HDL cholesterol, LDL cholesterol and triglyceride, BMI, blood pressure, cognitive function, stroke, coronary heart disease, congestive heart failure, cancer, and type 2 diabetes. 
As described previously [15], blood samples were collected between 2006 and 2009, and $\mathrm{HbA}_{1 \mathrm{c}}$ was measured using identical ion exchange-based high performance liquid chromatography with the Tosoh 2.2 Plus and after 2007 with the Tosch G7 Glycohemoglobin Analyzer (Tosoh Medics, San Francisco, CA 94080) at the Advanced Research and Diagnostics Laboratory, University of Minnesota. Fasting glucose was measured after an 8-h fast in serum by the Roche hexokinase method (Roche Diagnostics, Indianapolis, IN 46250) on a Roche Modular P Chemistry Analyzer (Roche Diagnostics Corporation). Fasting insulin was measured after an 8-h fast in serum on a Roche Elecsys 2010 Analyzer (Roche Diagnostics Corporation) using a sandwich immunoassay method (Roche Diagnostics, Indianapolis, IN 46250). Assays of average leukocyte telomere length were described by Lee et al. [16]. Briefly, Telomeres (T) and beta-globin control (S) were amplified using real-time PCR of $95{ }^{\circ} \mathrm{C}$ for 10 min denaturation, 34 cycles of $95^{\circ} \mathrm{C}$ for $15 \mathrm{~s}$ and $55^{\circ} \mathrm{C}$ for 120 s performed on the CFX384 thermocycler (BioRad, Richmond, CA). The leukocyte telomere length was calculated from $\mathrm{T} / \mathrm{S}$ ratio using the linear regression formula of $b p=(1,585 * \mathrm{~T} / \mathrm{S}$ ratio $)+3582$. Fasting total cholesterol was measured after an 8 -h fast in serum using a cholesterol oxidase method (Roche Diagnostics, Indianapolis, IN 46250) on a Roche Modular P Chemistry Analyzer.

Fasting HDL-cholesterol was measured after an 8-h fast directly in serum using the Roche HDL-Cholesterol 3rd generation direct method (Roche Diagnostics, Indianapolis, IN 46250) on a Roche Modular P Chemistry Analyzer. Fasting triglycerides were measured after an 8-h fast in serum using Triglyceride GB reagent (Roche Diagnostics, Indianapolis, IN 46250) on a Roche Modular P Chemistry Analyzer. LDL-cholesterol was calculated by the Friedewald equation using the measured results for total cholesterol, HDL-cholesterol, and triglycerides. This equation (LDL-cholesterol $=$ total cholesterol HDL - (triglycerides/5)) can be used to calculate LDLcholesterol when triglycerides are less than $400 \mathrm{mg} / \mathrm{dL}$. The corrected values for triglyceride and LDL cholesterol was calculated for individuals taking lipid lowering medications [17]. BMI was calculated as weight $(\mathrm{kg}) /$ height $(\mathrm{m})^{2}$. Sitting systolic and diastolic blood pressure was obtained by the average of three measures using an automated blood pressure machine (BP-tru BPM 300, VMS MedTech, Coquitlam, Canada). Pulse Pressure was calculated as systolic blood pressure minus diastolic blood pressure. These results were corrected for individuals taking blood pressure modifying medication as previously published [18]. General cognitive function including arithmetic, memory, and orientation was assessed using the mini-mental state examination (MMSE) or Folstein test [19]. Stroke or cerebrovascular accident includes self-reported stroke, transient ischemic attack (TIA), or mini-stroke. Coronary heart disease was defined as self-reported myocardial infarction, heart attack, coronary angioplasty, or coronary artery bypass grafting. Heart failure or congestive heart failure and cancer (including breast cancer, colon or rectal cancer, esophageal cancer, leukemia or lymphoma, lung cancer, pancreatic cancer, prostate cancer, etc.) were selfreported. Type 2 diabetes was defined as use of diabetes medications or fasting glucose $\geq 126 \mathrm{mg} / \mathrm{dl}$.

\section{Genome-wide SNP array genotyping}

Illumina Human Omni 2.5 v1 was used to perform whole genome SNP genotyping on each study participant by CIDR (www.cidr.jhmi.edu), and the resulting data has been submitted to dbGaP under accession number phs000397.v1.p1. Genome-wide SNP variants within targeted sequenced regions were used as controls for sequencing accuracy as described below.

\section{Pooled sequencing and indexed custom library preparation}

We have previously published the entire protocol for custom hybridization capture of multiplexed, indexed next generation sequencing $[20,27]$. Briefly, customized adapter, blocker, pre- and post-hybridization PCR amplification primers and all index sequences used are listed in Additional file 1: Table S5 of the same report. We performed pooled capture of 464 candidate genes (Additional file 1: Table S3) selected collectively by the LLFS investigators due to their published association with age-related phenotypes. Candidate gene resequencing was performed from individually indexed DNA samples from LLFS participants [https://longlifefamilystudy.wustl.edu/LLFS/Home.html]. The bait set was created using the Agilent eArray online tool [https:// earray.chem.agilent.com/earray/] for the Agilent SureSelect Custom DNA Capture [http://www.genomics.agilent.com] using $2 \mathrm{X}$ tiling, an exon-centered layout strategy, and eliminating probes that overlap standard repeat masked regions by 20 or more bases. In total, there were $2,500,709$ bases $(2.5 \mathrm{Mb})$ covered by baits in 6,966 distinct intervals.

\section{Sequencing}

Sequencing was performed in the Genome Technology Access Center at Washington University using the HiSeq 2000 platform, generating 101 bp paired-end reads. GWA and sequencing information for the LLFS participants is available in $\mathrm{dbGaP}$ using accession number phs000397.v1.p1.

\section{Sequencing alignment, variant calling and filtering}

Sequencing analysis for indexed captures of all LLFS participants followed previously reported methods (27; 
section entitled "Pooled indexed custom capture data analysis" for more details). Briefly, raw sequence data was aligned against the human genome (hg19/NCBI 37.0) using Novoalign (Novocraft, Inc.) and, from aligned reads, variants were called using samtools-0.1.18 mpileup at only the $2.5 \mathrm{Mb}$ of target sequence within our custom hybridization array.

The thresholds used for filtering called variants from sequencing data were determined by comparing common base positions shared with existing GWA data. For genelevel burden tests, variants were filtered for coverage $<5$ fold, quality score $<45$, call rate $<60 \%$, MAF $>0.01$, Mendel errors, and nonfunctional variants. Single variants were filtered for coverage $<5$-fold, quality score $<45$, call rate $<$ $60 \%$, and Mendel errors. For rare variants $(<2 \%$ minor allele frequency), these thresholds provided a sensitivity of $\geq 94.9 \%$ and specificity of $\geq 99.9 \%$ [27]. Mendel error cutoffs were implemented as follows: $\mathrm{MAF}=0$ to $<0.01$ for $>2$ pedigrees with the Mendel error; $\mathrm{MAF}=0.01$ to $<0.05$ for $\geq 7$ pedigrees; MAF $=0.05$ to $<0.1$ for $\geq 12$ pedigrees; $\mathrm{MAF}=0.1$ to $<0.2$ for $\geq 26$ pedigrees; $\mathrm{MAF}=0.2$ to $<0.3$ for $\geq 30$ pedigrees; $\mathrm{MAF}=0.3$ to $<0.4$ for $\geq 32$ pedigrees; $\mathrm{MAF}=0.4$ to $<0.51$ for $\geq 38$ pedigrees.

\section{Statistical analyses \\ Trait exceptionality scores for survival, BMI, blood pressure, blood lipid levels, blood glucose level, blood insulin level, cognitive function, type 2 diabetes, heart disease, cancer, and stroke}

The LLFS cohort is enriched with longer-lived and healthier individuals than the general population, as well as the Framingham Heart Study. To account for this difference and increase our statistical power for detecting sequence variants associated with aging-related phenotypes, such as survival, BMI, blood pressure, blood lipid levels, blood glucose level, blood insulin level, cognitive function, type 2 diabetes, heart disease, cancer, and stroke are transformed to "trait exceptionality scores". Higher trait exceptionality scores indicate that, for a given trait, an individual is significantly different from the reference population for the same trait, resulting in longer individual survival and/or better health. Using sex- and birth-year specific cohort life tables from the 2012 trustees report of the United States Social Security Administration, the trait exceptionality scores for survival were calculated for the oldest (proband) generation in LLFS, along with any blood relatives within the same generation regardless of age. These calculations are conditional on survival to age 40 and were determined as the negative logarithm of the probability of survival beyond age at last contact. Trait exceptionality scores for BMI, blood pressure, blood lipid levels, blood glucose level, blood insulin level, and cognitive function were also calculated as the negative logarithm of the probability of the trait score compared to the age/sex/ birth-cohort matched in the Framingham Heart Study. Trait exceptionality scores for type 2 diabetes, heart disease, cancer, and stroke were calculated as the negative logarithm of the probability of the onset age of the disease, and compared to the distribution of the age of onset for each trait in the Framingham Heart Study. There are two additional components to the HAI, which are pulmonary vital capacity and serum creatinine. However, these were not available in Framingham Heart Study and were not included in our analyses.

\section{Phenotype transformation and covariates adjustment}

To increase the power and reduce the confounder effects of our analyses, we performed the following covariates adjustment and transformation. $\mathrm{HbA}_{1 \mathrm{c}}$ was adjusted for age, age ${ }^{2}$, age ${ }^{3}$, field center and the top twenty principal components (PCs); the standardized residuals from a stepwise covariate adjustment were used as final phenotype for the following association analyses. The leukocyte telomere length was transformed using an inverse normal function. This transformed trait was adjusted for covariates age, sex, education, field center, smoking, alcohol consumption, marital status, history of heart disease, and twenty PCs (PC8 was the only significant $\mathrm{PC}$ associated with leukocyte telomere length in the multivariate polygenic model). Mortality weighted healthy aging index were adjusted for age, sex, and 10 PCs and the residuals were used in the following analyses. The residuals of trait exceptionality scores adjusted for field centers and 20 PCs were used in the following analyses.

\section{Single variant association testing}

Family relatedness was estimated based on pedigree structure using the "kinship" R package. Accounting for this relatedness within families as random effects, the additive genetic fixed effects of SNPs were analyzed using the linear mixed effects model implemented in the "Imekin" $\mathrm{R}$ packages [21, 22]. Q-Q plots for the phenotypes analyzed are shown in Additional file 1: Figure S1 and demonstrate that, compared to genomic control (calculated as median of observed $\mathrm{p}$ value / median of expected $\mathrm{p}$ value to avoid estimation bias), each is within 0.578-1.09 using this approach. Thus, the type I error rate is low in this study.

\section{Rare variant testing across genes}

Currently un-weighted sum score (UWSS) [23], weighted sum score (WSS) [24], P-value Weighted Sum Test (PWST) method [25] and family based sequence kernel association test (famSKAT) [26] are commonly used for estimating the effects of rare variants. Because the PWST and famSKAT are likely to have greater power 
to detect rare causal variants, we applied these two algorithms to assess the influence of called rare variants. This was necessary since standard association tests of individual rare variants $(\mathrm{MAF}<0.01)$ are underpowered unless sample sizes or effect sizes are very large, functional variants within the same gene based on bioinformatics annotation by ANNOVAR [27] were analyzed together in this study. For each group of variants, UWSS was calculated as the total number of variant alleles carried by a subject. The weight was calculated based on allele frequency in controls and WSS was calculated as the weighted sum of minor alleles over a group of variants. The UWSS or WSS score was treated as a single predictor variable $(X)$ and fit into a linear model. The kinship matrix was incorporated into the linear models to adjust for familial relatedness between subjects. Parameters in each model were estimated by the maximum likelihood algorithm and tested by the Wald test. Rather than using a fixed weight, the PWST method adaptively calculates the weights for individual variants from the observed genotype and phenotype data and then performs a permutation procedure for family data to avoid false positive inflation due to the over fitting of the adaptive score WSS.

\section{Results}

\section{Sample characteristics}

Phenotype

Our results included 4,217 LLFS subjects (mean of age 70.56 for men and 70.05 for women) with complete phenotypic and genotypic information. Individuals with inadequate GWA or sequencing coverage were excluded. The study participants tend to have healthier profiles compared with other cohorts of adults. For these individuals, the mean score of healthy aging index (3.64 in men and women) and mortality weighted healthy aging index (3.24 in men and 3.03 in women) are low. The average age of the LLFS probands and their relatives (95.8 in men and 99.4 in women) is much higher than the cohort life expectancy of US and Danish (an LLFS study site) early $20^{\text {th }}$ century birth cohorts (60-70 years) and the current life expectancy in both populations $(\sim 80$ years).

\section{Sequencing results}

To reduce false positive variant calls, stringent filtering of raw sequence data was employed and is described in Table 1. After filtering, 30,112 variants within 439 genes were identified with an average coverage of 47 -fold, quality score of 175 and call rate of 0.97 . As shown in Fig. 1, 37.4 \% $(11,261)$ of all called variants fell within coding regions with $22.4 \%(6,745)$ being either missense (non-synonymous SNV) or nonsense (stop-gain and stop-loss) variants; $50.7 \%(15,281)$ fell within regulatory
Table 1 Filter Applied in LLFS sequence data

Among 464 Candidate genes, 448 genes with 48,918 variants sequenced

\begin{tabular}{ll}
\hline Filter $1: \geq 5 \times$ coverage & $47 \pm 36$ \\
Filter $2: \geq 45$ quality score & $175 \pm 53$ \\
Filter $3: \geq 60 \%$ call rate & $97 \% \pm 7 \%$ \\
Filter 4: Mendel Errors & MAF dependent \\
439 genes with 30,112 variants analyzed & \\
\hline
\end{tabular}

5' or 3' untranslated regions (UTR5 or UTR3, respectively). The majority of variants $(59.8 \%)$ were found within a single LLFS family (Additional file 1: Table S4) and $(88.74 \%, n=26,723)$ were rare, occurring at $<1 \%$ in the general population, and $12,303(46.04 \%)$ were singletons (Additional file 1: Table S5).

\section{Single variant analyses}

Because there are correlations between the target phenotypes, the likelihood of a single variant having a functional effect on a given phenotype was Bonferroni corrected by dividing 0.05 by the total number of variants queried without accounting for the number of analyzed phenotypes. This resulted in $\mathrm{p}$-values of $7.56 \times 10^{-6}$ for survival and cancer and $1.47 \times 10^{-5}$ for HDL, LDL and triglycerides. We first attempted to determine if any single variant identified by sequencing was significantly associated with survival. We identified a novel regulatory variant in the 3' UTR of Oligonucleotide/Oligosaccharide-Binding Fold Containing 1, located on chromosome 10q24.33 (OBFC1; see Table 2) found in 13 individuals from six pedigrees (Additional file 1: Table S6), which was significantly associated $\left(p=6.18 \times 10^{-7}\right)$ with trait exceptionality for survival in long lived families. Intronic variants of $O B F C 1$ genotyped by Illumina array were also queried, and two rare variants (rs79250842 in 11 individuals from 5 pedigrees and rs77987791 in 5 individuals from 1 pedigree) were also found to have a significant association with survival (Fig. 2). OBFC1 is involved in telomere maintenance and falls within a recently reported LLFS family-based association peak for telomere length on chromosome 10 as shown in Fig. 2 [16]. This observation is supported by the association of the same OBFC1 intronic variant (rs77987791) with telomere length $(p=0.038)$. Interestingly, one long-lived pedigree (pedID 25609942) with 14 family members was clustered for all three rare variants in seven family members, which suggests that multiple rare variants within $O B F C 1$ might contribute to longer lifespan. The comparison of "survival" to OBFC1 genotype is shown in Additional file 1: Figure S2A.

Linear mixed model based single variant testing is not a stable method for rare variants with $<10$ minor allele 


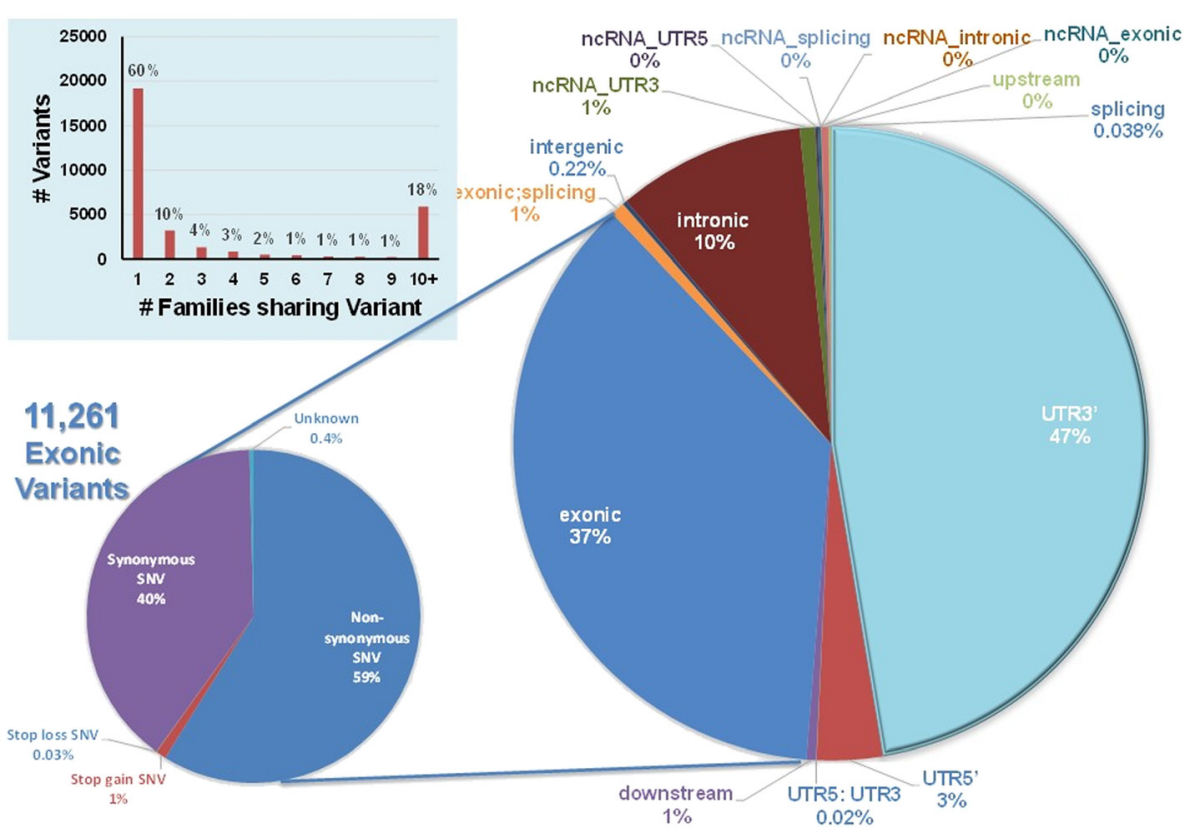

Fig. 1 The distribution of sequenced variants within different genomic region

copies [28], which is strengthened by our pedigree-based structure. While the effect of any single variant may be slight, we found a significant association with survival for 23 different variants in 23 healthy aging candidate genes (Table 3). Among these 23 variants, only four have been described in dbSNP. Eight of these genes (in bold) have published reports linking them to longer lifespan [9, 29-34]. These variants clustered in two families with longer survival (Fig. 3), suggesting that the aggregation of multiple variants in these genes may have a larger effect on survival. One subject who survived to age 110 carried 15 of these variants, and another surviving to age 101 years carries 9 of these variants. None of the variants were carried by spousal controls.
Among the 439 candidate genes, 160 are well-known human longevity related genes based on LongevityMap (http://genomics.senescence.info/longevity/) [35] and A Catalog of Published Genome-Wide Association Studies (http:/www.genome.gov/gwastudies/). When considering survival and any single variant with a p-value of $<0.05$ and more than 10 allelic copies in the LLFS cohort, we replicated 76 variants within these 160 genes (Additional file 1: Table S7), which supports the validity of our analysis model. Four variants within FOXO3, a well-known longevity related gene [36-39], were replicated for an association $(p<0.05)$ with survival.

We found associations with phenotypes other than survival. One candidate gene, IGF1R (Insulin-Like Growth

Table 2 Significant results of single variant testing for variants with more than 10 copies. Nine single variants with at least 10 allelic copies were identified within five phenotypes. There were 6,613 variants analyzed across 426 genes for survival and cancer and 3,389 variants analyzed across 408 genes for HDL, LDL and triglycerides

\begin{tabular}{|c|c|c|c|c|c|c|c|c|c|c|c|c|}
\hline Phenotype (TE Score) & $c h r$ & Position (hg19) & $r s / D$ & gene & Function & Ref & Variant & MAF & $N$ & Beta & SE & $P$-value \\
\hline Survival & 10 & 105642272 & & OBFC1 & UTR3 & $C$ & G & 0.002 & 810 & 3.58 & 0.71 & $6.2 \mathrm{E}-7$ \\
\hline Cancer & 15 & 99501295 & & $I G F 1 R$ & UTR3 & A & G & 0.007 & 4210 & 0.58 & 0.13 & $3.7 E-6$ \\
\hline \multirow[t]{3}{*}{ HDL Cholesterol } & 16 & 57005301 & rs1532625 & CETP & intron & $\mathrm{T}$ & C & 0.38 & 3053 & 0.19 & 0.03 & $2.4 \mathrm{E}-13$ \\
\hline & 16 & 57015091 & rs5880 & CETP & nsyn-exon & C & G & 0.047 & 4049 & -0.32 & 0.06 & $1.2 \mathrm{E}-8$ \\
\hline & 16 & 57017319 & rs1800777 & CETP & nsyn-exon & A & G & 0.029 & 3982 & -0.40 & 0.07 & $8.1 E-9$ \\
\hline \multirow[t]{3}{*}{ LDL Cholesterol } & 6 & 152679594 & rs62426382 & SYNE1 & syn-exon & G & A & 0.016 & 4035 & 0.45 & 0.09 & $1.6 \mathrm{E}-6$ \\
\hline & 19 & 45396144 & rs11556505 & TOMM40 & syn-exon & $\mathrm{T}$ & C & 0.10 & 4018 & -0.19 & 0.04 & $1.5 \mathrm{E}-6$ \\
\hline & 19 & 45397229 & rs1160983 & TOMM40 & syn-exon & A & G & 0.02 & 2686 & 0.53 & 0.10 & $1.2 \mathrm{E}-7$ \\
\hline Triglyceride & 11 & 116703640 & rs5128 & APOC3 & UTR3 & G & C & 0.09 & 4003 & -0.21 & 0.04 & $1.2 \mathrm{E}-7$ \\
\hline
\end{tabular}




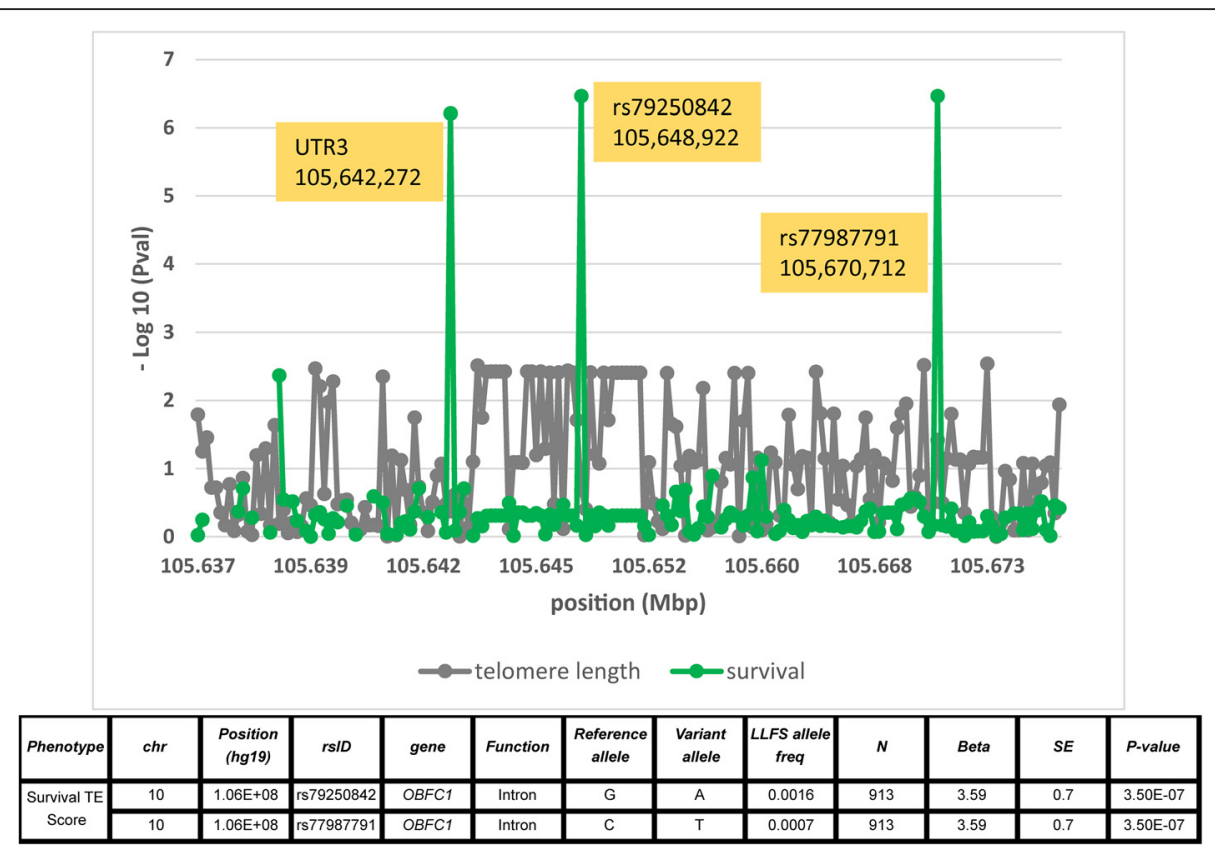

Fig. 2 Variant-wise association results on chromosome 10 around OBFC1 for telomere length phenotype (gray) and exceptional survival score (green) on chromosome 10 using both 1000 Genomes Project hybrid and candidate gene sequenced genotypes. Two additional rare variants from genome-wide array results showed significant association with survival exceptionality score. The association p-value of rs77987791 with telomere length is 0.038

Factor 1 Receptor, located on chromosome 15q26.3) is well known to be associated with cell growth and various cancers [40]. As shown in Table 1, we noted one new cancer associated rare UTR3 variant (chr15:99501295) in this gene.

In addition, four genes (CETP, APOC3, SYNE1 and TOMM40) were associated with blood lipid levels. The cholesterol ester transfer protein, CETP (located on chromosome 16q21), is essential for metabolism of plasma lipoproteins [41]. Three known HDL cholesterol related variants within CETP, one common intronic variant (rs1532625) [42] and two rare exonic variants (rs5880, rs1800777) [43], were replicated in our study. APOC3 (Apolipoprotein C3, located on chromosome 11q23.3) is critical for triglyceride metabolism and a potential therapeutic target for metabolic syndrome [44]. One known common triglyceride related variant (rs5128) [45] in the UTR3 region of $A P O C 3$ was replicated. LDL cholesterol was associated with one synonymous variant in SYNE1 (Spectrin Repeat Containing, Nuclear Envelope 1, on chromosome 6q25.2) and two synonymous variants in TOMM40 (Translocase Of Outer Mitochondrial Membrane 40 Homolog, on chromosome 19q13.32). TOMM40 has been identified in genome-wide screens for dyslipidemia and carotid artery disease [46, 47]. More importantly, TOMM40 rs10524523 polymorphism in combination with APOE alleles significantly influences late-onset Alzheimer's disease and longevity [48]. Comparisons for all variants listed in Table 2 between their associated phenotype and the variant genotype are shown in Additional file 1: Figure S2A-I.

\section{Analyses of multiple variants across genes}

To better characterize genes associated with our phenotypes due to multiple, pedigree-specific rare variants rather than more common single variants, we aggregated rare functional variants (exonic, UTR3, UTR5, splicing and non-coding RNA) within the same gene using the PWST [25] and famSKAT [26]. For association results, Bonferroni correction of 0.05 divided by the total number of genes resulted in a $p<1.14 \times 10^{-4}$ for significance and $p<1.0 \times 10^{-3}$ would merely be suggestive. With respect to survival, none of the candidate genes passed this significance threshold. When comparing our association results for survival to published longevity related genes, 20 genes were replicated with $\mathrm{p}$-value $<0.05$ (Additional file 1: Table S8).

We next performed PWSK and famSKAT analyses on our candidate genes to see if any gene harbored multiple putatively functional variants associated with the individual Healthy Aging Index (HAI) and those results are listed in Table 4. GSK3B (glycogen synthase kinase 3 beta, on chromosome 3q13.33) achieved significance by PWSK. GSK3B, a serine-threonine kinase, has been associated with Alzheimer's disease $[49,50]$ and may 
Table 3 Significant results of single variant testing for variants with less than 10 copies. Twenty-three rare variants with less than 10 minor allele copies were identified for a single phenotype (longevity). These variants clustered in two families with high trait exceptionality scores for longevity. Genes in bold have a previously published relationship to longevity [9, 29-34]

\begin{tabular}{|c|c|c|c|c|c|c|c|c|c|c|c|c|}
\hline$c h r$ & Position (hg19) & $r s / D$ & gene & Function & $\operatorname{Ref}$ & Variant & Minor Allele Count & MAF & $N$ & Beta & SE & $P$-value \\
\hline 7 & 17385235 & & $A H R$ & UTR3 & $A$ & G & 5 & 0.0006 & 810 & 3.58 & 0.71 & $6.18 \mathrm{E}-07$ \\
\hline 11 & 27680107 & rs8192466 & $B D N F$ & nsyn-exonic & A & G & 8 & 0.0009 & 810 & 5.02 & 1.01 & 7.41E-07 \\
\hline 16 & 57015065 & & CETP & intronic & $\mathrm{T}$ & $C$ & 3 & 0.0004 & 809 & 5.00 & 1.01 & 8.69E-07 \\
\hline 1 & 207813049 & & $C R 1$ & UTR3 & A & G & 5 & 0.0006 & 810 & 5.02 & 1.01 & 7.41E-07 \\
\hline 8 & 6735377 & & $D E F B 1$ & nsyn-exonic & $\mathrm{T}$ & $C$ & 1 & 0.0001 & 809 & 5.03 & 1.01 & $7.22 \mathrm{E}-07$ \\
\hline 10 & 71139772 & & $H K 1$ & nsyn-exonic & $\mathrm{T}$ & $C$ & 1 & 0.0001 & 794 & 5.04 & 1.01 & $6.53 \mathrm{E}-07$ \\
\hline 1 & 209959231 & & IRF6 & UTR3 & G & A & 4 & 0.0005 & 810 & 3.58 & 0.71 & $6.18 \mathrm{E}-07$ \\
\hline 21 & 35195906 & & ITSN1 & syn-exonic & A & $C$ & 1 & 0.0001 & 810 & 5.02 & 1.01 & 7.41E-07 \\
\hline 15 & 100256347 & & MEF2A & UTR3 & $C$ & T & 2 & 0.0002 & 810 & 5.02 & 1.01 & 7.41E-07 \\
\hline 1 & 12073445 & & MFN2 & UTR3 & $\mathrm{T}$ & $C$ & 3 & 0.0003 & 810 & 5.07 & 1.01 & $6.10 \mathrm{E}-07$ \\
\hline 4 & 100544175 & & MTTP & UTR3 & G & A & 1 & 0.0001 & 810 & 5.07 & 1.01 & $6.10 \mathrm{E}-07$ \\
\hline 2 & 1794502 & & MYT1L & UTR3 & $\mathrm{T}$ & $C$ & 1 & 0.0001 & 810 & 5.02 & 1.01 & 7.41E-07 \\
\hline 16 & 50267300 & & PAPD5 & UTR3 & $\mathrm{T}$ & $C$ & 4 & 0.0005 & 810 & 3.58 & 0.71 & $6.18 \mathrm{E}-07$ \\
\hline 2 & 223158616 & & PAX3 & UTR3 & $\mathrm{T}$ & $C$ & 3 & 0.0003 & 810 & 3.58 & 0.71 & $6.18 \mathrm{E}-07$ \\
\hline 7 & 95214257 & & PDK4 & UTR3 & G & A & 2 & 0.0002 & 810 & 5.07 & 1.01 & $6.10 \mathrm{E}-07$ \\
\hline 5 & 149206403 & rs374853976 & PPARGCIB & syn-exonic & A & G & 1 & 0.0001 & 714 & 5.07 & 1.00 & $4.72 \mathrm{E}-07$ \\
\hline 10 & 99130549 & & RRP12 & nsyn-exonic & $\mathrm{T}$ & $C$ & 1 & 0.0001 & 780 & 5.02 & 1.01 & 7.66E-07 \\
\hline 17 & 28525015 & rs199875985 & SLC6A4 & UTR3 & G & A & 1 & 0.0001 & 810 & 5.07 & 1.01 & $6.10 \mathrm{E}-07$ \\
\hline 15 & 67479818 & rs144245324 & SMAD3 & syn-exonic & $\mathrm{T}$ & $C$ & 3 & 0.0004 & 767 & 5.02 & 1.02 & $9.22 \mathrm{E}-07$ \\
\hline 3 & 24163883 & & THRB & UTR3 & $\mathrm{T}$ & $C$ & 7 & 0.0008 & 810 & 3.58 & 0.71 & $6.18 \mathrm{E}-07$ \\
\hline 14 & 81610971 & & $T S H R$ & UTR3 & A & G & 2 & 0.0002 & 810 & 5.07 & 1.01 & $6.10 \mathrm{E}-07$ \\
\hline 21 & 46189291 & & UBE2G2 & UTR3 & $\mathrm{T}$ & $C$ & 3 & 0.0004 & 810 & 5.02 & 1.01 & 7.41E-07 \\
\hline 19 & 9770038 & & ZNF562 & intronic & $\mathrm{T}$ & G & 2 & 0.0002 & 810 & 3.58 & 0.71 & $6.18 \mathrm{E}-07$ \\
\hline
\end{tabular}

regulate human aging via negative regulation of glucose homeostasis and Wnt signaling. In addition, NOTCH1 was significantly associated with diastolic blood pressure and TP53 with HDL (Table 4). NOTCH1 (chromosome $9 \mathrm{q} 34.3)$ is involved in a variety of developmental processes by controlling cell fate. In mice, NOTCH1 signaling is also required for vascular development [51]. TP53 (tumor protein P53, located on chromosome 17p13.1), is a well known tumor suppressor that regulates cell cycle and DNA repair. An inverse correlation of HDL cholesterol with cancer risk [52] might be explained by the association of TP53 with HDL cholesterol.

These results are likely an underestimate of significant or suggestive variants. Unfortunately, non-uniform hybridization to target loci due to local sequence context and the need for stringent filtering to reduce false positives resulted in gaps within the covered coding sequence of most genes, which could affect results due to false negatives. This is why the well-known longevity gene, ApoE [53], was not included in our analyses.

\section{Discussion}

The mechanisms driving healthy human aging are still mostly unclear. There is much debate on the evolutionary versus adaptive mechanisms of aging, such as programmed longevity, hormonal regulation of aging by insulin/IGF-1 signaling, programmed decline of the immune system, wear and tear theory, rate of oxygen basal metabolism, cross-linking theory and free radicals theory. These mechanisms are not inherently mutually exclusive and it is likely that aging is dictated by combinations of multiple mechanisms. Regardless, Kirkwood and Melev stated in a recent review that "it is obvious...that duration of life is dependent upon genotype" [54].

To take advantage of the unique family structure of the LLFS cohort, we have performed candidate gene resequencing to identify familial sequence variation that could explain multiple exceptional phenotypes contributing to long lifespan and healthy aging. Our results are limited by gaps in sequencing coverage of some genes due to non-uniform hybridization of baits across the entire cohort, which may mean an underestimate in the 


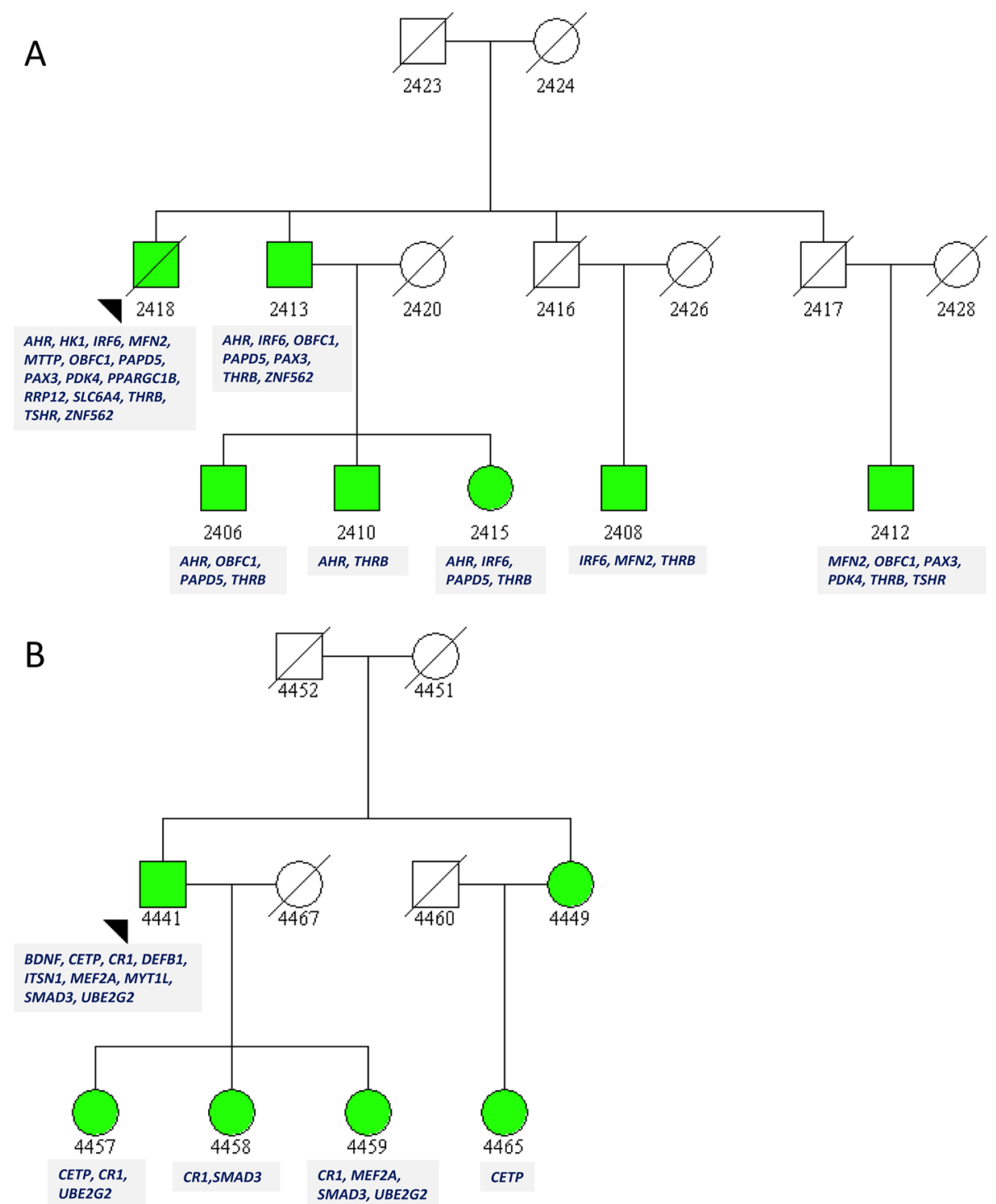

Fig. 3 Twenty-four rare variants within twenty-four genes clustered in two long-lived LLFS families (a) pedID 25609942 and (b) pedID 38652533. Individual 2418 and 4441 lived to 110 and 101 years of age, respectively. The longevity-associated genes harboring rare variants within these individuals (and their offspring) are listed below their symbol

number of familial variants in these genes. This strategy was previously used to identify rare familial variants in VEGFC (Vascular Endothelial Growth Factor C) by using linkage information in families with high LOD scores to inform targeted resequencing for rare variants. This experiment identified a familial variant in $V E G F C$ that explained $23.8 \%$ of phenotypic variance within a pedigree, but the same variant only described $0.1 \%$ of the phenotypic variance in unrelated individuals [55]. The same strategy was employed in the Insulin Resistance

Table 4 Collapsed rare variant analysis of candidate genes associated with HAl. These genes were found to have a $p$-value $<1.0 \times 10^{-3}$ by at least one burden-testing algorithm

\begin{tabular}{|c|c|c|c|c|c|c|c|c|c|}
\hline Phenotype & Gene & RefSeq & Total Exons & Analyzed Exons & Indiv Seq (\#) & Variants called (\#) & Ped (\#) & $P$-value (PWST) & $P$-value (Skat) \\
\hline $\begin{array}{l}\text { Healthy Aging Index } \\
\text { (Mortality Weighted) }\end{array}$ & GSK3B & NM_002093 & 12 & 8 & 3217 & 77 & 121 & $1.00 \mathrm{E}-4$ & $2.08 \mathrm{E}-4$ \\
\hline Diastolic Blood Pressure & NOTCHI & NM_017617 & 34 & 3 & 4112 & 55 & 78 & $1.00 \mathrm{E}-4$ & $5.37 \mathrm{E}-3$ \\
\hline HDL Cholesterol & TP53 & NM_000546 & 11 & 6 & 4073 & 21 & 86 & 1.00E-4 & 8.05E-3 \\
\hline
\end{tabular}


Atherosclerosis Family Study to identify a rare variant within the ADIPOQ (Adiponectin, C1Q And Collagen Domain Containing) gene describing $63 \%$ of the variance of plasma adiponectin levels, which are critical for glucose homeostasis [56]. Our results validate multiple SNPs and genes (OBFC1, CTEP, ZNF562) found associated with longevity and healthy survival. Using a sequencing-based approach to identify rare variants within pedigrees associated with exceptional phenotypes. Of note, we identified one rare, novel variant in $O B F C 1$ as well as two intronic variants from LLFS array data associated with survival. OBFC1 is involved in telomere maintenance; its role in promoting exceptionally healthy aging is intriguing given that this gene resides squarely within a family-based association peak for telomere length recently reported from the same LLFS families [16]. The idea that many longevityassociated variants can presumably work in synergy with an additive beneficial effect is supported by the observations from three LLFS families. One exceptionally long-lived LLFS family carries all three OBFC1 variants, while 23 additional beneficial rare variants are clustered in two other exceptionally long-lived LLFS families.

Because effect sizes from single rare variants are typically small, collapsing rare variants across larger loci (e.g. genes or pathways) can aggregate variants and highlight the importance of a larger genetic locus [25]. By analyzing our sequencing results in this fashion, we identified a more diverse set of genes associated (or suggestive of association) with additional healthy aging phenotypes. GSK3B, which is a kinase for over forty different proteins and plays key roles in numerous intracellular signaling pathways (cellular proliferation, migration, inflammation and immune response, glucose regulation, and apoptosis) [57] was identified in this analysis and may play an important role in healthy aging. This gene has been associated with a number of age-related diseases such as type II diabetes, Alzheimer's disease, inflammatory disorders, cancer and bipolar disorder [58].

Another healthy aging candidate gene, CETP, is a wellknown regulator of HDL and has been associated with healthy aging in Ashkenazi Jewish $[59,60]$ as well as Alzheimer's disease [61]. The negative association of several variants with HDL, LDL and triglycerides in our study indicates that harmful genetic variants still exist in the long-lived subjects. This paradoxical phenomenon might be due to antagonistic effects on the development of other age-related disorders, gene-age, gene-gene, or gene-environment interactions [62]. To understand these intersections, further investigation of age and environment specific effects of these lipid regulatory genes on multiple aging phenotypes is needed.

\section{Conclusions}

Under the Rare Variant/Complex Phenotype hypothesis, multiple rare variants have an aggregate effect on complex phenotypes or diseases, but identifying the genes and the relative contributions of the various sequence changes is difficult. By leveraging the pedigree structure and cohort size of the LLFS study, we demonstrate the utility of sequencing within pedigrees to identify inherited genetic variation influencing specific parameters of healthy aging. These data are limited by representing primarily genes that were already known to be associated with the various phenotypes being investigated. A larger survey of the genome in the LLFS cohort, especially the linkage peaks for multiple healthy aging phenotypes, will facilitate discovery of new genes and putative mechanisms for the genetic regulation of the complexities of aging.

\section{Ethics approval and consent to participate}

Each participant provided written, informed consent prior to inclusion in this study. This study is in compliance with the Helsinki Declaration and the results reported herein have been approved by the National Institute of Aging, the Human Research Protection Office of the coordinating center at Washington University under IRB\#201106316, the University of Pittsburgh Institutional Review Board, the Boston University Office of the Institutional Review Board, the Columbia University Institutional Review Board and the Regional Scientific Ethical Committees for Southern Denmark.

\section{Consent for publication \\ Not applicable.}

\section{Availability of data}

GWA and sequencing information for the LLFS participants is available in dbGaP using accession number phs000397.v1.p1.

\section{Additional file}

Additional file 1: Supplementary Materials. (PDF 1024 kb)

\section{Abbreviations}

LLFS: Long Life Family Study; HDL: high density lipoprotein; LDL: low density lipoprotein; BMI: body mass index; TIA: transient ischemic attack; MMSE: mini mental state examination; UTR: untranslated region; FLoSS: family longevity selection score; HAl: healthy aging index; GWAS: genome wide association study; PCs: principal components; MAF: minor allele frequency; $\mathrm{HgbA}_{1}$ : glycated hemoglobin; UWSS: un-weighted sum statistic; WSS: weighted sum statistic; PWST: P-value weighted sum test; famSKAT: family-based serial kernel association test.

Competing interests

The authors declare that they have no competing interests. 


\section{Authors' contributions}

All authors have read and approved the final manuscript. TED, BT, ABN, KC, RM and MAP designed the experimental plan. SEC, BTL prepared the sequencing libraries. TED, EIR, LW, SJL, JHL, QZ, EWD, HJA, MAP analyzed and interpreted data. TED, SJL, MAP drafted and revised the manuscript.

\section{Funding}

This work was supported by the National Institutes of Health, National Institute of Aging [U01 AG023746 (The Long Life Family Study)]. We would like to thank W. Rossi and E. Hadley for helpful discussions. We thank the Genome Technology Access Center in the Department of Genetics at Washington University School of Medicine for next-generation sequencing The Center is partially supported by NCl Cancer Center Support Grant P30 CA91842 to the Siteman Cancer Center and by ICTS/CTSA Grant UL1RR024992 from the National Center for Research Resources (NCRR), a component of the National Institutes of Health $(\mathrm{NIH})$, and NIH Roadmap for Medical Research. This publication is solely the responsibility of the authors and does not necessarily represent the official view of NCRR or $\mathrm{NIH}$.

\section{Author details}

${ }^{1}$ Center for Genome Sciences and Systems Biology, Washington University School of Medicine, 660 South Euclid Avenue, Campus Box 8116, St. Louis, MO 63108, USA. ${ }^{2}$ Department of Pediatrics, Washington University School of Medicine, 660 South Euclid Avenue, Campus Box 8116, St. Louis, MO 63108, USA. ${ }^{3}$ Division of Statistical Genomics, Department of Genetics, Washington University School of Medicine, St. Louis, MO, USA. ${ }^{4}$ Sergievsky Center, College of Physicians and Surgeons, Columbia University New York, New York, NY, USA. ${ }^{5}$ Taub Institute, College of Physicians and Surgeons, Columbia University New York, New York, NY, USA. ${ }^{6}$ Department of Epidemiology, School of Public Health, Columbia University New York, New York, NY, USA. ${ }^{7}$ Department of Laboratory Medicine and Pathology, University of Minnesota, Minneapolis, MN, USA. ${ }^{8}$ Department of Epidemiology, University of Pittsburgh Graduate School of Public Health, Pittsburgh, PA, USA. ${ }^{9}$ The Danish Aging Research Center, Epidemiology, University of Southern Denmark, Odense, Denmark. ${ }^{10}$ Gertrude H. Sergievsky Center and the Taub Institute for Research on Alzheimer's Disease and the Aging Brain, Columbia University, New York City, NY, USA.

\section{Received: 7 May 2015 Accepted: 4 April 2016}

Published online: 09 April 2016

\section{References}

1. Herskind AM, McGue M, Holm NV, Sorensen TI, Harvald B, Vaupel JW. The heritability of human longevity: a population-based study of 2872 Danish twin pairs born 1870-1900. Hum Genet. 1996;97:319-23.

2. Schachter F, Faure-Delanef L, Guenot F, Rouger H, Froguel P, Lesueur-Ginot L, Cohen D. Genetic associations with human longevity at the APOE and ACE loci. Nat Genet. 1994;6:29-32.

3. van Bockxmeer FM. ApoE and ACE genes: impact on human longevity. Nat Genet. 1994;6:4-5.

4. Christensen $\mathrm{K}$, Johnson TE, Vaupel JW. The quest for genetic determinants of human longevity: challenges and insights. Nat Rev Genet. 2006;7:436-48.

5. Deelen J, Beekman M, Uh HW, Helmer Q, Kuningas M, Christiansen L, Kremer D, 500 van der Breggen R, Suchiman HE, Lakenberg N, et al. Genome-wide association study identifies a single major locus contributing to survival into old age; the APOE locus revisited. Aging Cell. 2011;10:686-98.

6. Bonafe M, Barbieri M, Marchegiani F, Olivieri F, Ragno E, Giampieri C, Mugianesi E, Centurelli M, Franceschi C, Paolisso G. Polymorphic variants of insulin-like growth factor I (IGF-I) receptor and phosphoinositide 3-kinase genes affect IGF-I plasma levels and human longevity: cues for an evolutionarily conserved mechanism of life span control. J Clin Endocrinol Metab. 2003;88:3299-304.

7. Willcox BJ, Donlon TA, He Q, Chen R, Grove JS, Yano K, Masaki KH, Willcox DC, Rodriguez B, Curb JD. FOXO3A genotype is strongly associated with human longevity. Proc Natl Acad Sci USA. 2008;105:13987-92.

8. Pawlikowska L, Hu D, Huntsman S, Sung A, Chu C, Chen J, Joyner AH, Schork NJ, Hsueh WC, Reiner AP, et al. Association of common genetic variation in the insulin/IGF1 signaling pathway with human longevity. Aging Cell. 2009:8:460-72.
9. Sebastiani P, Solovieff N, Dewan AT, Walsh KM, Puca A, Hartley SW, Melista E, Andersen S, Dworkis DA, Wilk JB, et al. Genetic signatures of exceptional longevity in humans. PLoS One. 2012;7:e29848.

10. Sebastiani P, Sun FX, Andersen SL, Lee JH, Wojczynski MK, Sanders JL, Yashin A, Newman AB, Perls TT. Families Enriched for Exceptional Longevity also have Increased Health-Span: Findings from the Long Life Family Study. Front Public Health. 2013;1:38.

11. Newman AB, Glynn NW, Taylor CA, Sebastiani P, Perls TT, Mayeux R, Christensen K, Zmuda JM, Barral S, Lee JH, et al. Health and function of participants in the Long Life Family Study: A comparison with other cohorts. Aging (Albany NY). 2011;3:63-76.

12. Barral S, Cosentino S, Costa R, Andersen SL, Christensen K, Eckfeldt JH, Newman AB, Perls TT, Province MA, Hadley EC, et al. Exceptional memory performance in the Long Life Family Study. Neurobiol Aging. 2013;34:2445-8.

13. Sanders JL, Minster RL, Barmada MM, Matteini AM, Boudreau RM, Christensen K, Mayeux R, Borecki IB, Zhang Q, Perls T, et al. Heritability of and mortality prediction with a longevity phenotype: the healthy aging index. J Gerontol A Biol Sci Med Sci. 2014;69:479-85.

14. Sebastiani P, Hadley EC, Province M, Christensen K, Rossi W, Perls T, Ash AS A family longevity selection score: ranking sibships by their longevity, size, and availability for study. Am J Epidemiol. 2009;170:1555-62.

15. An P, Miljkovic I, Thyagarajan B, Kraja AT, Daw EW, Pankow JS, Selvin E, Kao WH, Maruthur NM, Nalls MA, et al. Genome-wide association study identifies common loci influencing circulating glycated hemoglobin ( $\mathrm{HbA})$ levels in non-diabetic subjects: The Long Life Family Study (LLFS). Metabolism. 2013;63:461-8.

16. Lee JH, Cheng R, Honig LS, Feitosa M, Kammerer CM, Kang MS, Schupf N, Lin SJ, Sanders JL, Bae H, et al. Genome wide association and linkage analyses identified three loci-4q25, 17q23.2, and 10q11.21-associated with variation in leukocyte telomere length: the Long Life Family Study. Front Genet. 2013;4:310.

17. Wu J, Province MA, Coon H, Hunt SC, Eckfeldt JH, Arnett DK, Heiss G, Lewis CE, Ellison RC, Rao DC, et al. An investigation of the effects of lipid-lowering medications: genome-wide linkage analysis of lipids in the HyperGEN study. BMC Genet. 2007;8:60.

18. Wu J, Kraja AT, Oberman A, Lewis CE, Ellison RC, Arnett DK, Heiss G, Lalouel JM, Turner ST, Hunt SC, Province MA, Rao DC. A summary of the effects of antihypertensive medications on measured blood pressure. Am J Hypertens. 2005; 18:935-42.

19. Folstein MF, Folstein SE, McHugh PR. "Mini-mental state". A practical method for grading the cognitive state of patients for the clinician. J Psychiatr Res. 1975;12:189-98.

20. Ramos E, Levinson BT, Chasnoff S, Hughes A, Young AL, Thornton K, Li A, Vallania FL, Province M, Druley TE. Population-based rare variant detection via pooled exome or custom hybridization capture with or without individual indexing. BMC Genomics. 2012;13:683.

21. Pinheiro JC, Bates DB. Mixed-effects models in S and S-Plus. New York, NY: Springer; 2000.

22. Abrahantes JC, Burzykowski T. A version of the EM algorithm for proportional hazard model with random effects. Biom J. 2005;47:847-62.

23. Li B, Leal SM. Methods for detecting associations with rare variants for common diseases: application to analysis of sequence data. Am J Hum Genet. 2008;83:311-21.

24. Madsen BE, Browning SR. A groupwise association test for rare mutations using a weighted sum statistic. PLoS Genet. 2009;5:e1000384.

25. Zhang Q, Irvin MR, Arnett DK, Province MA, Borecki I. A data-driven method for identifying rare variants with heterogeneous trait effects. Genet Epidemiol. 2011;35:679-85.

26. Chen $H$, Meigs JB, Dupuis J. Sequence kernel association test for quantitative traits in family samples. Genet Epidemiol. 2013;37:196-204.

27. Wang K, Li M, Hakonarson H. ANNOVAR: functional annotation of genetic variants from high-throughput sequencing data. Nucleic Acids Res. 2010;38: e164.

28. Lee S, Abecasis GR, Boehnke M, Lin X. Rare-variant association analysis:study designs and statistical tests. Am J Hum Genet. 2014;95:5-23.

29. Dato S, Crocco P, D'Aquila P, de Rango F, Bellizzi D, Rose G, Passarino G. et al. Exploring the role of genetic variability and lifestyle in oxidative stress response for healthy aging and longevity. Int J Mol Sci. 2013;14 16443-72.

30. Atzmon G, Rincon M, Schechter CB, Shuldiner AR, Lipton RB, Bergman A, Barzilai N. Lipoprotein genotype and conserved pathway for exceptional longevity in humans. PLoS Biol. 2006;4(4):e113. 
31. Trahan G. Mitochondrial-nuclear epistasis: implications for human aging and longevity. Ageing Res Rev. 2011;10:238-52.

32. Geesaman BJ, Benson E, Brewster SJ, Kunkel LM, Blanche H, Thomas G, et al. Haplotype-based identification of a microsomal transfer protein marker associated with the human lifespan. Proc Natl Acad Sci USA. 2003;100:14115-20.

33. Gondo $Y$, Hirose $N$, Arai $Y$, Yamamura $K$, Shimizu K, Takayama M, Ebihara $Y$, Nakazawa S, Inagaki H, Masui $Y$, et al. Contribution of an affect-associated gene to human longevity: prevalence of the long-allele genotype of the serotonin transporter-linked gene in Japanese centenarians. Mech Ageing Dev. 2005:126:1178-84.

34. Atzmon G, Barzilai N, Surks Ml, Gabriely I. Genetic predisposition to elevated serum thyrotropin is associated with exceptional longevity. J Clin Endocrinol Metab. 2009:94:4768-75.

35. Budovsky A, Craig T, Wang J, Tacutu R, Csordas A, Lourenco J, Fraifeld VE, de Magalhaes JP. LongevityMap: a database of human genetic variants associated with longevity. Trends Genet. 2013;29:559-60.

36. Li Y, Wang WJ, Cao H, Lu J, Wu C, et al. Genetic association of FOXO1A and FOXO3A with longevity trait in Han Chinese populations. Hum Mol Genet. 2009;18:4897-904.

37. Broer L, Buchman AS, Deelen J, Evans DS, Faul JD, Lunetta KL, Sebastiani P, Smith JA, Smith AV, Tanaka T, Yu L, Arnold AM, Aspelund T, Benjamin EJ, De Jager PL, Eirkisdottir G, Evans DA, Garcia ME, Hofman A, Kaplan RC, Kardia SL, Kiel DP, Oostra BA, Orwoll ES, Parimi N, Psaty BM, Rivadeneira F, Rotter Jl, Seshadri S, Singleton A, Tiemeier H, Uitterlinden AG, Zhao W, Bandinelli S, Bennett DA, Ferrucci L, Gudnason V, Harris TB, Karasik D, Launer LJ, Perls TT, Slagboom PE, Tranah GJ, Weir DR, Newman AB, van Duijn CM, Murabito JM. GWAS of Longevity in CHARGE Consortium Confirms APOE and FOXO3 Candidacy. J Gerontol A Biol Sci Med Sci. 2015;70:110-8.

38. Soerensen M, Dato S, Christensen K, McGue M, Stevnsner T, Bohr VA, Christiansen L. Replication of an association of variation in the FOXO3A gene with human longevity using both case-control and longitudinal data. Aging Cell. 2010;9:1010-7.

39. Zeng Y, Cheng L, Chen H, Cao H, Hauser ER, Liu Y, Xiao Z, Tan Q, Tian XL, Vaupel JW. Effects of FOXO genotypes on longevity: a biodemographic analysis. J Gerontol A Biol Sci Med Sci. 2010;65:1285-99.

40. Haisa M. The type 1 insulin-like growth factor receptor signalling system and targeted tyrosine kinase inhibition in cancer. J Int Med Res. 2013:41:253-64

41. Rader DJ, deGoma EM. Future of cholesteryl ester transfer protein inhibitors. Annu Rev Med. 2014;65:385-403.

42. Reilly D, Hao K, Jensen MK, Girman CJ, Rimm EB. Use of systems biology approaches to analysis of genome-wide association studies of myocardial infarction and blood cholesterol in the nurses' health study and health professionals' follow-up study. PLoS One. 2013;8:e85369.

43. Ridker PM, Pare G, Parker AN, Zee RY, Miletich JP, Chasman DI. Polymorphism in the CETP gene region, HDL cholesterol, and risk of future myocardial infarction: Genomewide analysis among 18245 initially healthy women from the Women's Genome Health Study. Circ Cardiovasc Genet. 2009;2:26-33.

44. van Dijk KW, Rensen PC, Voshol PJ, Havekes LM. The role and mode of action of apolipoproteins CIII and AV: synergistic actors in triglyceride metabolism? Curr Opin Lipidol. 2004;15:239-46.

45. Smith CE, Tucker KL, Scott TM, Van Rompay M, Mattei J, Lai CQ, Parnell LD, Junyent M, Lee YC, Garcia-Bailo B, et al. Apolipoprotein C3 polymorphisms, cognitive function and diabetes in Caribbean origin Hispanics. PLoS One. 2009;4:e5465.

46. Ronald J, Rajagopalan R, Ranchalis JE, Marshall JK, Hatsukami TS, Heagerty PJ, Jarvik GP. Analysis of recently identified dyslipidemia alleles reveals two loci that contribute to risk for carotid artery disease. Lipids Health Dis. 2009;8:52.

47. Talmud PJ, Drenos F, Shah S, Shah T, Palmen J, Verzilli C, Gaunt TR, Pallas J, Lovering R, Li K, et al. Gene-centric association signals for lipids and apolipoproteins identified via the HumanCVD BeadChip. Am J Hum Genet. 2009;85:628-42

48. Maruszak A, Peplonska B, Safranow K, Chodakowska-Zebrowska M, Barcikowska M, Zekanowski C. TOMM40 rs10524523 polymorphism's role in late-onset Alzheimer's disease and in longevity. J Alzheimers Dis. 2012:28:309-22.

49. Kwok JB, Loy CT, Hamilton G, Lau E, Hallupp M, Williams J, Owen MJ, Broe GA, Tang N, Lam L, Powell JF, Lovestone S, Schofield PR. Glycogen synthase kinase-3 beta and tau genes interact in Alzheimer's disease. Ann Neurol 2008:64:446-54

50. Forlenza OV, Torres CA, Talib LL, de Paula VJ, Joaquim HP, Diniz BS, Gattaz WF. Increased platelet GSK3B activity in patients with mild cognitive impairment and Alzheimer's disease. J Psychiatr Res. 2011;45:220-4.

51. Dill MT, Rothweiler S, Djonov V, Hushchuk R, Tornillo L, Terracciano L, MeiliButz S, Radtke F, Heim MH, Semela D. Disruption of Notch1 induces vascular remodeling, intussusceptive angiogenesis, and angiosarcomas in livers of mice. Gastroenterology. 2012;142:967-77.

52. Zhao W, Guan J, Horswell R, Li W, Wang Y, Wu X, Hu G. HDL Cholesterol and Cancer Risk Among Patients With Type 2 Diabetes. Diabetes Care 2014;37:3196-203

53. Kulminski AM, Arbeev KG, Culminskaya I, Arbeeva L, Ukraintseva SV, Stallard E, Christensen K, Schupf N, Province MA, Yashin Al. Age, gender, and cancer but not neurodegenerative and cardiovascular diseases strongly modulate systemic effect of the Apolipoprotein E4 allele on lifespan. PLoS Genet. 2014;10:e1004141.

54. Kirkwood TB, Melev S. On the programmed/non-programmed nature of ageing within the life history. Curr Biol. 2011;21:R701-7.

55. Shi G, Simino J, Rao DC. Enriching rare variants using family-specific linkage information. BMC Proc. 2011;Suppl 9:S82.

56. Bowden DW, An SS, Palmer ND, Brown WM, Norris JM, Haffner SM, Hawkins GA, Guo X, Rotter Jl, Chen YD, et al. Molecular basis of a linkage peak: exome sequencing and family-based analysis identify a rare genetic variant in the ADIPOQ gene in the IRAS Family Study. Hum Mol Genet. 2010:19:4112-20.

57. Jope RS, Johnson GV. The glamour and gloom of glycogen synthase kinase-3. Trends Biochem Sci. 2004;29:95-102.

58. Schaffer BA, Bertram L, Miller BL, Mullin K, Weintraub S, Johnson N, Bigio EH, Mesulam M, Wiedau-Pazos M, Jackson GR, et al. Association of GSK3B with Alzheimer disease and frontotemporal dementia. Arch Neurol. 2008;65:1368-74.

59. Atzmon G, Rincon M, Rabizadeh P, Barzilai N. Biological evidence for inheritance of exceptional longevity. Mech Ageing Dev. 2005;26:341-5.

60. Barzilai N, Atzmon G, Schechter C, Schaefer EJ, Cupples AL, Lipton R, Cheng S, Shuldiner AR. Unique lipoprotein phenotype and genotype associated with exceptional longevity. JAMA. 2003;290:2030-40.

61. Chen JJ, Li YM, Zou WY, Fu JL. Relationships between CETP genetic polymorphisms and Alzheimer's disease risk: a meta-analysis. DNA Cell Biol. 2014;33:807-15

62. Ukraintseva S, Yashin A, Arbeev K, Kulminski A, Akushevich I, Wu D, Joshi G, Land, KC, Stallard,E (2015) Puzzling role of genetic risk factors in human longevity:"risk alleles" as pro-longevity variants. Biogerontology. [Epub ahead of print]

\section{Submit your next manuscript to BioMed Central and we will help you at every step:}

- We accept pre-submission inquiries

- Our selector tool helps you to find the most relevant journal

- We provide round the clock customer support

- Convenient online submission

- Thorough peer review

- Inclusion in PubMed and all major indexing services

- Maximum visibility for your research

Submit your manuscript at www.biomedcentral.com/submit
C Biomed Central 